\title{
Motor imagery training improves precision of an upper limb movement in patients with hemiparesis
}

\author{
Luzia Grabherr ${ }^{\mathrm{a}, \mathrm{b}, *}$, Corinne Jola $^{\mathrm{c}}$, Gilberto Berra ${ }^{\mathrm{d}}$, Robert Theiler ${ }^{\mathrm{d}}$ and Fred W. Mast ${ }^{\mathrm{a}}$ \\ ${ }^{a}$ Department of Psychology, University of Bern, Bern, Switzerland \\ ${ }^{\mathrm{b}}$ School of Health Sciences, University of South Australia, Adelaide, Australia \\ ${ }^{\mathrm{c}}$ Division of Social and Health Sciences - Psychology, Abertay University, Dundee, UK \\ ${ }^{\mathrm{d}}$ Department of Rheumatology, Physical Medicine and Rehabilitation, Triemli City Hospital, Zurich, Switzerland
}

\begin{abstract}
.
BACKGROUND: In healthy participants, beneficial effects of motor imagery training on movement execution have been shown for precision, strength, and speed. In the clinical context, it is still debated whether motor imagery provides an effective rehabilitation technique in patients with motor deficits.

OBJECTIVE: To compare the effectiveness of two different types of movement training: motor imagery vs. motor execution. METHODS: Twenty-five patients with hemiparesis were assigned to one of two training groups: the imagery or the executiontraining group. Both groups completed a baseline test before they received six training sessions, each of which was followed by a test session. Using a novel and precisely quantifiable test, we assessed how accurately patients performed an upper limb movement.

RESULTS: Both training groups improved performance over the six test sessions but the improvement was significantly larger in the imagery group. That is, the imagery group was able to perform more precise movements than the execution group after the sixth training session while there was no difference at the beginning of the training.

CONCLUSIONS: The results provide evidence for the benefit of motor imagery training in patients with hemiparesis and thus suggest the integration of cognitive training in conventional physiotherapy practice.
\end{abstract}

Keywords: Mental practice, rehabilitation, physical therapy, stroke

\section{Introduction}

Explicit motor imagery is commonly referred to as the conscious mental rehearsal of a movement without actually executing the action. It has been proposed that

\footnotetext{
*Address for correspondence: Luzia Grabherr, School of Health Sciences, University of South Australia, City East Campus, GPO Box 2471, Adelaide, SA 5001, Australia. Tel.: +61 88302 1295; E-mail: luzia.grabherr@unisa.edu.au.
}

motor imagery corresponds to a subliminal activation of the motor system (Jeannerod \& Frak, 1999) and it has been repeatedly shown that motor imagery is, in many critical aspects, closely related to action execution (Decety, 1996; Lotze \& Halsband, 2006; Mast, Bamert, \& Newby, 2007). One line of evidence supporting a link between imagined and executed actions comes from studies revealing similarities in the time it takes to imagine performing a particular action (e.g. 
tying a shoe) and the time it takes to actually perform the action (Decety \& Jeannerod, 1995; Papaxanthis, Schieppati, Gentili, \& Pozzo, 2002). Mentally practicing a specific movement can improve speed, accuracy, and strength of motor execution (Gentili, Papaxanthis, \& Pozzo, 2006; Yaguez et al., 1998; Yue \& Cole, 1992). These findings suggest that motor imagery involves some of the same mechanisms engaged during the execution of actual movements. Neuroimaging studies have shown that areas involved in motor execution also play a role in motor imagery (Munzert, Lorey, \& Zentgraf, 2009). Lafleur and colleagues (2002), for example, demonstrated that repeated imagery of foot movements is accompanied by changes in brain activation over time similar to those that occur when the movements are actually executed. Moreover, imagined and executed movements elicit activation in the corresponding contralateral motor cortices (Ehrsson, Geyer, \& Naito, 2003; Wolbers, Weiller, \& Buchel, 2003).

These findings on motor imagery have an applied impact of high relevance, for example in sport psychology (Blasing et al., 2012; Holmes \& Calmels, 2008; Moran, Guillot, Macintyre, \& Collet, 2012) or rehabilitation (Jackson, Lafleur, Malouin, Richards, \& Doyon, 2001; Warner \& McNeill, 1988). However, in order to use motor imagery successfully in a clinical setting the question remains whether patients with motor deficits are still capable of mentally simulating movements they can no longer execute. If the capability to mentally rehearse the movement were no longer possible, motor imagery training would most likely not result in an improved motor performance. Interestingly, findings suggest that motor imagery abilities can recover after stroke (de Vries, Tepper, Otten, \& Mulder, 2011) or that motor imagery abilities remain intact in hemiplegic patients after years of limb disuse (Johnson, 2000; Johnson, Sprehn, \& Saykin, 2002; Johnson-Frey, 2004). Nevertheless, there are also critical voices (Olsson \& Nyberg, 2010) and it has to be pointed out that motor imagery training is cognitively demanding requiring patients to be in an alert state.

During the last decade, motor imagery training has indeed been proposed as a promising rehabilitation practice in patients with hemiparesis. Numerous case and small group studies report benefits in gait (Dickstein, Dunsky, \& Marcovitz, 2004; Dunsky, Dickstein, Ariav, Deutsch, \& Marcovitz, 2006; Jackson, Doyon, Richards, \& Malouin, 2004; Kim \& Lee, 2013; Oh, Kim, Kim, Yoo, \& Jeon, 2010) and upper limb mobility (Crosbie, McDonough, Gilmore, \& Wiggam,
2004; Dijkerman, Ietswaart, Johnston, \& MacWalter, 2004; Page, Levine, \& Hill, 2007; Page, Levine, \& Leonard, 2005; Page, Levine, Sisto, \& Johnston, 2001; Page, Levine, Sisto, \& Johnston, 2001; Stevens \& Stoykov, 2003; Yoo, Park, \& Chung, 2001). Moreover, results of controlled experimental studies using larger patient samples are promising. They report evidence of motor imagery training for transfer effects to untrained motor tasks (Liu, Chan, Lee, \& Hui-Chan, 2004; Liu et al., 2009), immediate improvement after only one training session (Malouin, Richards, Doyon, Desrosiers, \& Belleville, 2004) as well as long lasting effects (Page, Murray, Hermann, \& Levine, 2011). It is not without some disappointment that the study with the largest sample size to date (a total of 121 stroke patients were tested from which 39 participated in the motor imagery training group) and a well-controlled research design fell short of providing evidence for the efficacy of motor imagery training (Ietswaart et al., 2011). The number of reviews on the use of motor imagery training in rehabilitation is astonishing, offering a good overview and critical discussions (Barclay-Goddard, Stevenson, Poluha, \& Thalman, 2011; S. Braun et al., 2013; Braun, Beurskens, Borm, Schack, \& Wade, 2006; de Vries \& Mulder, 2007; Dickstein \& Deutsch, 2007; Malouin \& Richards, 2010; Sharma, Pomeroy, \& Baron, 2006; Sterr \& Conforto, 2012). One common conclusion is the need for yet more sound empirical evidence.

Despite cognitive-therapeutic exercises with a motor imagery component that were described early on (Perfetti \& Rossetto, 1997) and a critical amount of clinical research, motor imagery training is by far not exploited and used on a regular basis (Malouin, Jackson, \& Richards, 2013). Conventional rehabilitation trainings use movement repetition (we will refer to such kind of therapies as execution trainings) to improve motor performance. Since patients with hemiparesis encounter severe difficulties to move their limbs and conventional therapies depend on the movement ability of the impaired $\operatorname{limb}(\mathrm{s})$, the benefit of the rehabilitation is strongly inflicted by the patients' state. Hence, if imagery abilities are still reminiscent despite severely reduced movement capabilities, imagery training may be an effective rehabilitation technique. We thus investigated whether repeated imagined movements of the impaired upper limb improve motor performance by evaluating and comparing the effectiveness of two different types of rehabilitation training: mental imagery (IMAG) vs. execution (EXEC). 
Table 1

Overview of demographic and clinical variables of the patients

\begin{tabular}{lccc}
\hline & & & Between-group differences \\
& IMAG group & EXEC group & 12 \\
\hline Patients (n) & 13 & $8 \mathrm{~m}, 4 \mathrm{f}$ & \\
Gender (male, female) & $8 \mathrm{~m}, 5 \mathrm{f}$ & $68.9 \pm 14.7$ & \\
Age & $61.3 \pm 15.3$ & 12 stroke & \\
Cause cerebral lesion & 10 stroke, 3 other & $61,6 \mathrm{r}$ & \\
Lateralization of lesion (left, right) & $101,3 \mathrm{r}$ & $27 \pm 55$ & $t(11.6)=-1.266, p=0.22$ \\
Time from onset lesion to treatment (months) & $6 \pm 9=0.22$ \\
Number of therapy appointments & $4.5 \pm 1.2$ & $4.5 \pm 1.3$ & $t(23)=0.077, p=0.94$ \\
Total duration of training (days) & $13 \pm 8$ & $11 \pm 4$ & $t(23)=0.777, p=0.45$ \\
\hline
\end{tabular}

Mean and standard deviations are indicated for patients of the mental imagery (IMAG) and the motor execution (EXEC) group. In 22 out of the 25 patients a stroke was at the origin of their hemiparesis. Brain tumors (two patients) and a vascular encephalopathy (one patient) were the other causes. Most patients ( 8 in the IMAG and 8 in the EXEC group) began training less than three months after stroke onset. However, some patients with chronic hemiparesis were also included in the study. Namely, one patient in the EXEC group had a stroke 16 years ago explaining the large mean and standard deviation of the EXEC group. Independent samples $t$-tests were calculated to assess possible group differences. However, the groups did not significantly differ in any of the tested variables.

\section{Methods}

\subsection{Participants}

Hospitalized patients with hemiparesis were informed about the study and the possibility to participate. Please note that the training received during this study was in addition to their regular physiotherapy. Informed consent was obtained prior to the first training session. The study was approved by the local ethic committees and has been performed in accordance with the Declaration of Helsinki. In total 25 patients with hemiparesis (9 women and 16 men) aged between 37 and 92 (mean age of 65) were included in the study. All patients were right-handed according to a shortened version of the Edinburgh Inventory of Handedness (Oldfield, 1971).

The patients were assessed with an extensive clinical and cognitive questionnaire that lasted around $45 \mathrm{~min}$ utes. Clinical factors tested were hemiparetic status (i.e. severity of hemiparesis), ideomotor apraxia, sensory body perception in both hands and arms (proprioception and exteroception) and neglect. The latter was assessed with manual and tactile line bisection tests as well as visual (dual pictures) and imagery tests (describing a place). Cognitive abilities tested were language, mental rotation, short-term memory, spatial orientation, and attention. The minimum requirements they had to meet were sufficient cognitive reserve to understand the instructions (especially verbal understanding) and to have good postural control (they had to be able to sit at a table). There was no criteria concerning age, affected side, severity or cause of hemiparesis for inclusion in the study. After its completion, the patients were allocated either to the mental imagery (IMAG) or the motor execution (EXEC) group. To ensure that the two groups consisted of an equal amount of patients and matched concerning two potential prognostic factors, age and gender, we choose not to use a true randomization process. Instead a minimisation procedure (dynamic allocation) was adopted (Altman \& Bland, 2005). Table 1 gives an overview of the patients' demographic and clinical variables.

\subsection{Apparatus}

We specifically designed an apparatus (Fig. 1) to be able to easily but precisely and quantifiably measure

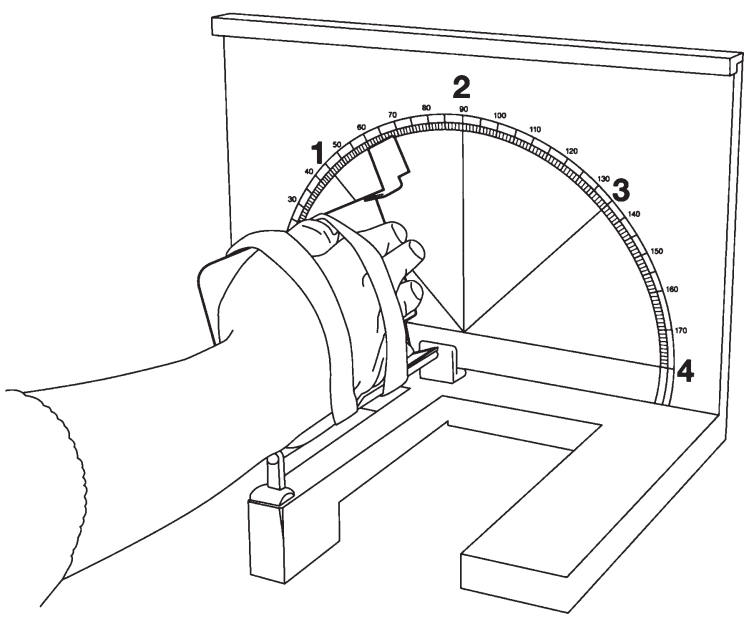

Fig. 1. Experimental apparatus. The apparatus was designed to be able to measure hand position precisely and objectively. It consisted of a wooden platform, a rotatable aluminium clamp and a background illustration indicating the positions (0-4) as well as the degrees. 
upper limb performance. The movement involved pronation and supination of the forearm. We chose to focus on this movement because it is critical for activities of daily living and in rehabilitation this movement often receives particular attention. The patients were sitting next to the experimenter with the apparatus positioned on a table in front of them. Their affected hand was put in a rotatable clamp keeping their fingers aligned. The patients then received a short introduction to get acquainted with the apparatus. They learnt to memorize five different positions each corresponding to a given number $(0,1,2,3,4)$. Five equidistant markers on the backboard of the apparatus indicated these different positions: $0=0^{\circ}$ (start position; pronation: horizontal hand position with the back of the hand facing upward), $1=45^{\circ}, 2=90^{\circ}$ (the hand is perpendicular to the surface of the table), $3=135^{\circ}$ degrees, and $4=180^{\circ}$ (supination: horizontal position with the back of the hand facing downward). The experimenter manually guided the patient's hand (always starting from pronation going towards supination) so that they could memorize how each position felt. The tip of the clamp served as a pointer, indicating the exact position of the hand (resolution of the scale $1^{\circ}$ ). Thus, the patients received visual (they could see the backboard with indications of the positions) and proprioceptive (guidance of their hand) feedback to familiarize themselves with the apparatus.

\subsection{Training}

Before each training session, a short state assessment was carried out in order to evaluate the patient's condition at the given point in time. The experimenter checked the verbal understanding, the postural control and the awareness of the patient. We also recorded how many and what kind of therapies the patient already had that day. In case the patient lacked verbal understanding, postural control or awareness (e.g. when the patient was very tired), training was postponed to another day.

The training consisted either of the imagery or the execution training as described below, depending on the allocation. All patients received six standardized training sessions, during which they were blindfolded.

Mental Imagery Training: The mental imagery training corresponded to an internal, kinaesthetic, firstperson perspective training ("feel" the hand moving as opposed to "see" the hand moving). In each training session, 16 positions had to be imagined. Each position was tested as follows: In a first step, the patients were read a number aloud by the experimenter (e.g. position 3). They had to imagine moving their affected hand to this position without actually executing the movement. They reported when they had mentally reached the position. In a second step, the experimenter moved the patient's hand to a position, that either matched or mismatched the imagined position and the patient had to judge whether it matched or not. Hence, a response was correct when the patient either correctly identified a matching position or rejected an incongruent position. A response was incorrect, when the patient either judged an incongruent position as correct or did not recognize a matching position. If an incongruent position was correctly rejected, the experimenter moved the hand to another position and the patient had to judge again whether the hand was now at the correct (the previously imagined) position or not. When the hand was moved to the target position and the answer was correct, the experimenter gave a verbal feedback "yes, this is position 3, the position you had to imagine moving your hand to" and the trial ended. If the patient did not recognize the matching position, the experimenter gave the feedback "no, this is actually the correct position, position 3 , the position you had to imagine moving your hand to", and the trial also ended. In case the patient judged an incongruent position as correct, the experimenter guided the patient's hand to the correct (the previously imagined) position in order to provide corrective feedback. After the feedback the next trial began. Each trial began from the start position 0 . After 16 imagined positions (each position had to be mentally reached four times) a test session as described below followed. The sequence of all positions was predefined and randomized.

Motor Execution Training: This type of training represents the more common procedure used in rehabilitation. The patients were also read a number aloud by the experimenter (e.g. position 3) but instead of imagining moving their hand to this given position, the patients were instructed to try to actually execute the movement. The experimenter manually guided the hand if necessary. The patients had to stop the movement in case they could move the hand themselves or else to say "stop" when they thought to have reached the indicated position. Each trial started from position 0 and was repeated once. A training session of the EXEC group consisted of 32 trials. Please note that there were 16 trials per training session in the IMAG group. This is in order to make the duration of a training session equally long, because one trial lasted about twice as long in IMAG. Otherwise, the protocol was the same for the IMAG and the EXEC group. In fact, the training protocols were 
identical for corresponding patients (e.g. patient 1 in the IMAG group performed the same order of positions as patient 1 in the EXEC group). This is also true for the test protocols: the same randomized order of positions was used for corresponding patients. One training session lasted approximately 20 to 30 minutes. The number of appointments during which these training sessions were completed could vary from patient to patient depending on their physical and mental state. Some patients felt comfortable to complete more than one training session per day. The two groups did not differ in the overall number of appointments (Table 1) and an appointment never lasted more than 45 minutes in total.

\subsection{Tests}

Prior to the first training (baseline) and after each training session, performance was tested with the following two tasks. Patients were blindfolded and the tests were the same for both groups:

Recognition task: The experimenter moved the patient's hand to one of the four possible positions (1-4) and the patient had to guess the position. This task is very similar to the second part of the training of the IMAG group (first part is the imagination of the movement). Responses were recorded on a protocol sheet. The dependent variable is the error rate (amount of false responses/total number of trials).

Movement task: The experimenter indicated a number (e.g. position 4) and the patient was instructed to try to reach this position as accurately as possible. The experimenter assisted the movement if necessary. The patient had to say "stop" when he believed to have reached the indicated position or to stop the movement in case they could move the hand themselves. The experimenter pointed out that he will not guide the hand to the correct end position but continue the movement over the entire range of $180^{\circ}$. This task corresponds to the training of the EXEC group. The end position reached by the patient was recorded on a protocol sheet. The dependent variable is the deviation between the target position and the actual end position (measured in degrees).

Per test (i.e. recognition task and movement task), each position was repeated twice, except for the baseline test where each position was tested only once. The order of the tasks was counterbalanced across patients. In total, each patient conducted a baseline test, six training and six test sessions.

\subsection{Statistical analysis}

To assess possible group differences of the variables measured during the assessment, either independent sample $t$-tests or Mann-Whitney U tests were calculated, depending on the type of variable. Error rates (recognition task) and mean deviation (movement task) were analysed using repeated-measure analyses of variance (ANOVAs) with the variable test (test 1 , test 6 ) as within-subjects factor and training group (IMAG, EXEC) as between-subjects factor. Significant effects were further analysed using post-hoc tests (Bonferroni). Statistical tests were performed with SPSS 17.0.

\section{Results}

The IMAG and the EXEC group did not significantly differ on the variables that were measured with the assessment questionnaire. These variables were hemiparetic status $(U=73.5, p=0.806)$, ideomotor apraxia $(U=56.5, p=0.203)$, sensory body perception $(U=56.5, p=0.263)$ and cognitive tests: mental rotation $(t(23)=1.109, p=0.279)$, short-term memory $(t(23)=-0.317, p=0.754)$, spatial orientation $(t$ $(20)=1.127, p=0.273)$, and attention $(t(13)=1.096$, $p=0.293$ ). Five patients (three in the IMAG group, two in the EXEC group) showed signs of neglect in the assessment questionnaire and/or were previously diagnosed with neglect.

\subsection{Recognition task}

Figure 2 illustrates the results achieved in the recognition task of the IMAG and the EXEC group from baseline testing to test session 6 .

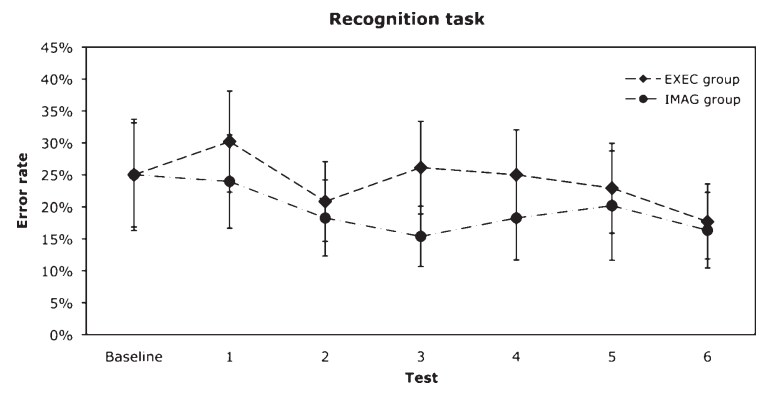

Fig. 2. Results recognition task. Mean error rates and standard errors of the mean (SEM) are shown for the IMAG and the EXEC group. The error rate decreased significantly in both groups from baseline testing to test session 6 . Thus, their performance improved in the recognition task. 
The IMAG and the EXEC group showed a significant decrease in error rates over the test sessions $\left(F(1,22)=10.796, p<0.01, \eta_{p}{ }^{2}=0.329\right)$. There was neither a significant difference between the two groups $\left(F(1,22)=0.446, p=0.511, \eta_{p}^{2}=0.020\right)$ nor a significant test $\times$ group interaction effect $(F(1,22)=0.020$, $\left.p=0.888, \eta_{p}{ }^{2}=0.001\right)$. These findings suggest that both groups improved equally well in recognition performance.

\subsection{Movement task}

Figure 3 illustrates the results achieved in the movement task of the IMAG and the EXEC group from baseline testing to test session 6 .

The IMAG and the EXEC group showed a significant decrease in mean deviation over the test sessions $\left(F(1,23)=19.895, p<0.001, \eta_{p}^{2}=0.464\right)$. There was no significant main effect of the factor group ( $F$ $\left.(1,23)=2.241, p=0.148, \eta_{p}{ }^{2}=0.089\right)$. Interestingly, however, the interaction test $\times$ group was significant $\left(F(1,23)=5.410, p<0.05, \eta_{p}^{2}=0.190\right)$. Post -hoc tests revealed, that the IMAG group was able to perform more precise movements than the EXEC group after the sixth training session $(p<0.01)$ whereas there was no such difference after the first training session $(p=0.969)$. These findings suggest that while both groups improved in performance, the IMAG group profited more from their training than the EXEC group.

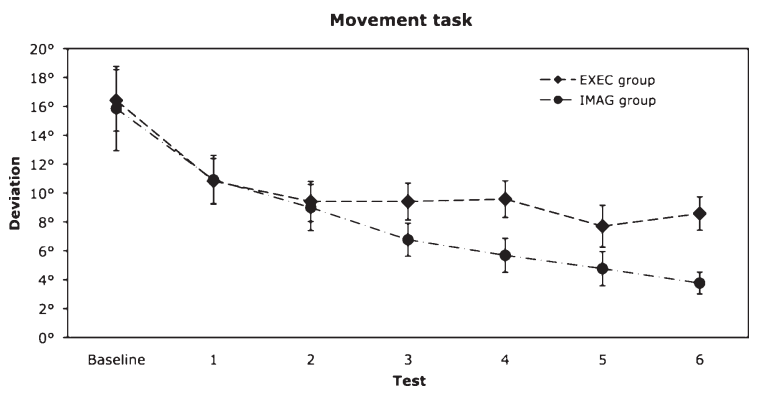

Fig. 3. Results movement task. Mean deviations (in degrees) and standard errors of the mean (SEM) are shown for the IMAG and the EXEC group. Mean deviation from target location decreased significantly in both groups from baseline testing to test session 6 . Thus, their performance improved in the movement task. More importantly, the improvement of the IMAG group in test session 6 was significantly greater than the average improvement achieved by the EXEC group.

\section{Discussion}

While the EXEC group was trained to execute the movement of the upper limb, the IMAG group practiced to mentally simulate the movement. Results in the recognition (error rate) and the movement task (accuracy of the hand end position) showed that patients of both training groups improved in the ability to correctly perceive the position of their affected hand and to accurately move their hand to a given position. Importantly, both groups started off with the same performance levels but the movement task reveals that the improvement was greater in the IMAG group compared to the EXEC group.

How can we explain the finding that the IMAG group performed more precise movements than the EXEC group at the end of the training? Our study was designed in such a way that both groups - in their specific training method - participated in an equal amount of training sessions. Hence, we argue that the differences are not due to differences in the amount of training. Neither can the effect be explained by differences in demographic and clinical variables nor by variables measured with the assessment questionnaire (e.g. hemiparetic status, short-term memory). Thus, we suggest that the outcome difference observed between the two groups is due to the functional difference of the trainings themselves. These findings are clinically relevant. First, they are in line with previous studies using similar training groups (motor imagery vs. execution training) (Liu et al., 2004, 2009). Second, they suggest that mental imagery training can be an effective means to improve motor rehabilitation in patients with hemiparesis.

Notably, the mental imagery training differs from the execution training by means of an explicit focus on imagined movements as well as the involvement of passive movements. During the second part of the imagery training the experimenter moved the patient's hand to one of the four positions and the patient had to guess whether this position matched or mismatched the imagined number. This was a means to provide the patients with a feedback on their mental performance and to assure indirectly that they comply with the motor imagery instruction. Passive movements may have contributed to a certain extent to the success of the imagery training (Szameitat, Shen, Conforto, \& Sterr, 2012). Likely more important is the fact that these trainings were administered in addition to regular physiotherapy provided by the hospital, which involved execution training but not imagery training. Moreover, during the movement task all patients were required 
to perform overt movements. Thus, the IMAG group likely profited more from the training because it added a new component - motor imagery - to their regular training. This assumption is supported by other findings. Previous studies (Dijkerman et al., 2004; Page, Levine, \& Leonard, 2007; Page et al., 2005) - with the exception of a study lacking therapist and patient compliance (Bovend'Eerdt, Dawes, Sackley, Izadi, \& Wade, 2010) - reported that stroke patients who received conventional execution training combined with motor imagery training improved more than did those who received execution training alone. These and other studies are critically discussed in a recent review in terms of different modes of motor imagery training delivery. An important framework is provided to help guide researchers and clinicians to implement motor imagery training and address critical issues like compliance, amount of training and interaction of physical and mental practice (Malouin et al., 2013).

While neural activation is overlapping, it is not completely identical for executed and imagined actions (Mast et al., 2007; Munzert et al., 2009). Thus, a training that relies on both - executed and imagined actions most likely broadens the range of brain areas implicated in the rehabilitation process. Our results suggest that motor execution has its limits and with the addition of motor imagery outcomes can be improved. Especially in severe cases where patients have little or no motor control it can be useful to start with motor imagery before commencing execution training. In this way motor imagery can serve as a "gateway" to (constraintinduced) movement therapy (Page, Levine, \& Hill, 2007). Moreover, from a patient's perspective motor imagery training can be more motivating than common motor execution training, notably in such severe cases. In fact, we had patients who reported that they were very comfortable with the motor imagery training because they felt they had more control and can actually "do something". Similar positive verbal reports from patients after a motor imagery training intervention have been reported recently (Dickstein et al., 2014). It is also possible that some patients performed unsupervised motor imagery outside the scope of this study. We did not control for this and it would be difficult to do so. Essentially, the possibility for patients to perform motor imagery training on their own is a great asset. The use of computer-assisted motor imagery trainings has been proposed, making it possible to reduce the patient's need for skilled support. This enables the patient to practice more often and thus to possibly enhance the outcome of the training (Gaggioli, Meneghini,
Morganti, Alcaniz, \& Riva, 2006). Moreover, recent studies successfully applied a brain computer interface design using motor imagery to enhance motor recovery after stroke (Bai, Huang, Fei, \& Kunz, 2014; Caria et al., 2011; Varkuti et al., 2013).

Given the finding that the IMAG group showed improved performance in the movement task, why was there no such difference in the recognition task? Such a finding could have been expected since the recognition task resembled the training of the IMAG group and the movement task resembled the training of the EXEC group. We propose that no group differences were found in the recognition task because this test was too easy for some patients (ceiling effect) and not sensitive enough to show any group differences. Thus, a fine-grained measure allowing the detection of small changes - as was used in the movement task - seems critical. Possibly, the lack of sufficient test sensitivity may explain why some studies failed to show beneficial effects of motor imagery training.

It is of clinical relevance to know whether patients differ in their level of benefit from motor imagery training. Mutsaarts, Steenbergen \& Bekkering (2007) suggest that patients with left hemispheric brain damage have impaired motor imagery abilities while another study suggests the opposite (Malouin, Richards, \& Durand, 2012). Yet other studies report specific motor imagery deficits in the stroke-affected hand after both left and right hemispheric damage (Daprati, Nico, Duval, \& Lacquaniti, 2010; Sabate, Gonzalez, \& Rodriguez, 2004; Stinear, Fleming, Barber, \& Byblow, 2007) but propose a more dominant role of the left hemisphere for motor planning (Sabate et al., 2004). We could show a substantial improvement through motor imagery even though the IMAG group comprised ten patients with left-hemispheric lesions (and three with right-hemispheric lesions). Future studies should address this issue in detail to elaborate under which conditions mental imagery can or cannot unfold its beneficial effects.

In summary, a novel paradigm that allows for precisely assessing training outcomes was used to compare the effectiveness of motor imagery vs. motor execution training in patients with hemiparesis. The accuracy-measurement in the movement task provides evidence for an advantage in the rehabilitation of patients with hemiparesis through the addition of motor imagery training and thus suggests the integration of cognitive training in conventional physiotherapy practice. 


\section{Acknowledgments}

We thank Walter Schmid and Henri Gossweiler for constructing the apparatus, Ruth Möckli and the Rehaklinik Bellikon for their help in recruiting and testing patients and the Department of Neurology, University Hospital Zurich for helping constructing the assessment questionnaire. This research was supported by the Swiss National Science Foundation (Grant 611-066052 and PDFM1-114406, postdoctoral fellowship awarded to L.G.).

\section{Conflict of interest}

The authors declare no conflict of interest.

\section{References}

Altman, D. G., \& Bland, J. M. (2005). Treatment allocation by minimisation. BMJ, 330(7495), 843. doi: 10.1136/bmj.330.7495.843

Bai, O., Huang, D., Fei, D. Y., \& Kunz, R. (2014). Effect of real-time cortical feedback in motor imagery-based mental practice training. NeuroRehabilitation, 34(2), 355-363. doi: 10.3233/NRE-131039

Barclay-Goddard, R. E., Stevenson, T. J., Poluha, W., \& Thalman, L. (2011). Mental practice for treating upper extremity deficits in individuals with hemiparesis after stroke. Cochrane Database Syst Rev, (5), CD005950. doi: 10.1002/14651858.CD005950.pub4

Blasing, B., Calvo-Merino, B., Cross, E. S., Jola, C., Honisch, J., \& Stevens, C. J. (2012). Neurocognitive control in dance perception and performance. Acta Psychologica, 139(2), 300-308. doi: 10.1016/j.actpsy.2011.12.005

Bovend'Eerdt, T. J., Dawes, H., Sackley, C., Izadi, H., \& Wade, D. T. (2010). An integrated motor imagery program to improve functional task performance in neurorehabilitation: A single-blind randomized controlled trial. Arch Phys Med Rehabil, 91(6), 939946. doi: 10.1016/j.apmr.2010.03.008

Braun, S., Kleynen, M., van Heel, T., Kruithof, N., Wade, D., \& Beurskens, A. (2013). The effects of mental practice in neurological rehabilitation; a systematic review and meta-analysis. Frontiers in Human Neuroscience, 7, 390. doi: 10.3389/fnhum.2013.00390

Braun, S. M., Beurskens, A. J., Borm, P. J., Schack, T., \& Wade, D. T. (2006). The effects of mental practice in stroke rehabilitation: A systematic review. Archives of Physical Medicine and Rehabilitation, 87(6), 842-852. doi: 10.1016/j.apmr.2006.02.034

Caria, A., Weber, C., Brotz, D., Ramos, A., Ticini, L. F., Gharabaghi, A., Birbaumer, N., et al. (2011). Chronic stroke recovery after combined $\mathrm{BCI}$ training and physiotherapy: A case report. Psychophysiology, 48(4), 578-582. doi: 10.1111/j.14698986.2010.01117.x

Crosbie, J. H., McDonough, S. M., Gilmore, D. H., \& Wiggam, M. I. (2004). The adjunctive role of mental practice in the rehabilitation of the upper limb after hemiplegic stroke: A pilot study. Clinical Rehabilitation, 18(1), 60-68.

Daprati, E., Nico, D., Duval, S., \& Lacquaniti, F. (2010). Different motor imagery modes following brain damage. Cortex, 46(8), 1016-1030. doi: 10.1016/j.cortex.2009.08.002

de Vries, S., \& Mulder, T. (2007). Motor imagery and stroke rehabilitation: A critical discussion. Journal of Rehabilitation Medicine, 39(1), 5-13. doi: 10.2340/16501977-0020

de Vries, S., Tepper, M., Otten, B., \& Mulder, T. (2011). Recovery of motor imagery ability in stroke patients. Rehabilitation Research and Practice, 2011, 283840. doi: 10.1155/2011/283840

Decety, J. (1996). The neurophysiological basis of motor imagery. Behav Brain Res, 77(1-2), 45-52.

Decety, J., \& Jeannerod, M. (1995). Mentally simulated movements in virtual reality: Does Fitts's law hold in motor imagery? Behavioural Brain Research, 72(1-2), 127-134.

Dickstein, R., \& Deutsch, J. E. (2007). Motor imagery in physical therapist practice. Physical Therapy, 87(7), 942-953. doi: 10.2522/ptj.20060331

Dickstein, R., Dunsky, A., \& Marcovitz, E. (2004). Motor imagery for gait rehabilitation in post-stroke hemiparesis. Physical Therapy, 84(12), 1167-1177.

Dickstein, R., Levy, S., Shefi, S., Holtzman, S., Peleg, S., \& Vatine, J. J. (2014). Motor imagery group practice for gait rehabilitation in individuals with post-stroke hemiparesis: A pilot study. NeuroRehabilitation, 34(2), 267-276. doi: 10.3233/NRE-131035

Dijkerman, H. C., Ietswaart, M., Johnston, M., \& MacWalter, R. S. (2004). Does motor imagery training improve hand function in chronic stroke patients? A pilot study. Clinical Rehabilitation, 18(5), 538-549.

Dunsky, A., Dickstein, R., Ariav, C., Deutsch, J., \& Marcovitz, E. (2006). Motor imagery practice in gait rehabilitation of chronic post-stroke hemiparesis: Four case studies. International Journal of Rehabilitation Research, 29(4), 351-356.

Ehrsson, H. H., Geyer, S., \& Naito, E. (2003). Imagery of voluntary movement of fingers, toes, and tongue activates corresponding body-part-specific motor representations. Journal of Neurophysiology, 90(5), 3304-3316.

Gaggioli, A., Meneghini, A., Morganti, F., Alcaniz, M., \& Riva, G. (2006). A strategy for computer-assisted mental practice in stroke rehabilitation. Neurorehabilitation and Neural Repair, 20(4), 503-507.

Gentili, R., Papaxanthis, C., \& Pozzo, T. (2006). Improvement and generalization of arm motor performance through motor imagery practice. Neuroscience, 137(3), 761-772.

Holmes, P., \& Calmels, C. (2008). A neuroscientific review of imagery and observation use in sport. Journal of Motor Behavior, 40(5), 433-445. doi: 10.3200/JMBR.40.5.433-445

Ietswaart, M., Johnston, M., Dijkerman, H. C., Joice, S., Scott, C. L., MacWalter, R. S., \& Hamilton, S. J. (2011). Mental practice with motor imagery in stroke recovery: Randomized controlled trial of efficacy. Brain, 134(Pt 5), 1373-1386. doi: 10.1093/brain/awr077

Jackson, P. L., Doyon, J., Richards, C. L., \& Malouin, F. (2004). The efficacy of combined physical and mental practice in the learning of a foot-sequence task after stroke: A case report. Neurorehabilitation and Neural Repair, 18(2), 106-111.

Jackson, P. L., Lafleur, M. F., Malouin, F., Richards, C., \& Doyon, J. (2001). Potential role of mental practice using motor imagery in neurologic rehabilitation. Archives of Physical Medicine and Rehabilitation, 82(8), 1133-1141. 
Jeannerod, M., \& Frak, V. (1999). Mental imaging of motor activity in humans. Current Opinion in Neurobiology, 9(6), 735-739.

Johnson, S. H. (2000). Imagining the impossible: Intact motor representations in hemiplegics. Neuroreport, 11(4), 729-732.

Johnson, S. H., Sprehn, G., \& Saykin, A. J. (2002). Intact motor imagery in chronic upper limb hemiplegics: Evidence for activity-independent action representations. Journal of Cognitive Neuroscience, 14(6), 841-852.

Johnson-Frey, S. H. (2004). Stimulation through simulation? Motor imagery and functional reorganization in hemiplegic stroke patients. Brain and Cognition, 55(2), 328-331.

Kim, J. H., \& Lee, B. H. (2013). Action observation training for functional activities after stroke: A pilot randomized controlled trial. NeuroRehabilitation, 33(4), 565-574. doi: 10.3233/NRE130991

Lafleur, M. F., Jackson, P. L., Malouin, F., Richards, C. L., Evans, A. C., \& Doyon, J. (2002). Motor learning produces parallel dynamic functional changes during the execution and imagination of sequential foot movements. Neuroimage, 16(1), 142-157.

Liu, K. P., Chan, C. C., Lee, T. M., \& Hui-Chan, C. W. (2004). Mental imagery for promoting relearning for people after stroke: A randomized controlled trial. Archives of Physical Medicine and Rehabilitation, 85(9), 1403-1408.

Liu, K. P., Chan, C. C., Wong, R. S., Kwan, I. W., Yau, C. S., Li, L. S., \& Lee, T. M. (2009). A randomized controlled trial of mental imagery augment generalization of learning in acute poststroke patients. Stroke, 40(6), 2222-2225. doi: 10.1161/STROKEAHA.108.540997

Lotze, M., \& Halsband, U. (2006). Motor imagery. Journal of Physiology Paris, 99(4-6), 386-395.

Malouin, F., Jackson, P. L., \& Richards, C. L. (2013). Towards the integration of mental practice in rehabilitation programs. A critical review. Frontiers in Human Neuroscience, 7, 576. doi: 10.3389/fnhum.2013.00576

Malouin, F., \& Richards, C. L. (2010). Mental practice for relearning locomotor skills. Physical Therapy, 90(2), 240-251. doi: 10.2522/ptj.20090029

Malouin, F., Richards, C. L., Doyon, J., Desrosiers, J., \& Belleville, S. (2004). Training mobility tasks after stroke with combined mental and physical practice: A feasibility study. Neurorehabilitation and Neural Repair, 18(2), 66-75.

Malouin, F., Richards, C. L., \& Durand, A. (2012). Slowing of motor imagery after a right hemispheric stroke. Stroke Research and Treatment, 2012, 297217. doi: 10.1155/2012/297217

Mast, F. W., Bamert, L., \& Newby, N. (2007). Mind over Matter? Imagined body movements and their neuronal correlates. In F. W. Mast \& L. Jäncke (Eds.), Spatial Processing in Navigation, Imagery and Perception, (pp. 353-368), New York: Springer.

Moran, A., Guillot, A., Macintyre, T., \& Collet, C. (2012). Reimagining motor imagery: Building bridges between cognitive neuroscience and sport psychology. Br J Psychol, 103(2), 224247. doi: 10.1111/j.2044-8295.2011.02068.x

Munzert, J., Lorey, B., \& Zentgraf, K. (2009). Cognitive motor processes: The role of motor imagery in the study of motor representations. Brain Research Reviews, 60(2), 306-326. doi: 10.1016/j.brainresrev.2008.12.024

Mutsaarts, M., Steenbergen, B., \& Bekkering, H. (2007). Impaired motor imagery in right hemiparetic cerebral palsy. Neuropsychologia, 45(4), 853-859.
Oh, D. W., Kim, J. S., Kim, S. Y., Yoo, E. Y., \& Jeon, H. S. (2010). Effect of motor imagery training on symmetrical use of knee extensors during sit-to-stand and stand-to-sit tasks in post-stroke hemiparesis. NeuroRehabilitation, 26(4), 307-315. doi: 10.3233/NRE-2010-0567

Oldfield, R. C. (1971). The assessment and analysis of handedness: The Edinburgh inventory. Neuropsychologia, 9(1), 97-113.

Olsson, C. J., \& Nyberg, L. (2010). Motor imagery: If you can't do it, you won't think it. Scandinavian Journal of Medicine \& Science in Sports, 20(5), 711-715. doi: 10.1111/j.1600-0838.2010.01101.x

Page, S. J., Levine, P., \& Hill, V. (2007). Mental practice as a gateway to modified constraint-induced movement therapy: A promising combination to improve function. American Journal of Occupational Therapy, 61(3), 321-327.

Page, S. J., Levine, P., \& Leonard, A. (2007). Mental practice in chronic stroke: Results of a randomized, placebo-controlled trial. Stroke, 38(4), 1293-1297.

Page, S. J., Levine, P., \& Leonard, A. C. (2005). Effects of mental practice on affected limb use and function in chronic stroke. Archives of Physical Medicine and Rehabilitation, 86(3), 399-402.

Page, S. J., Levine, P., Sisto, S., \& Johnston, M. V. (2001). A randomized efficacy and feasibility study of imagery in acute stroke. Clinical Rehabilitation, 15(3), 233-240.

Page, S. J., Levine, P., Sisto, S. A., \& Johnston, M. V. (2001). Mental practice combined with physical practice for upper-limb motor deficit in subacute stroke. Physical Therapy, 81(8), 1455-1462.

Page, S. J., Murray, C., Hermann, V., \& Levine, P. (2011). Retention of motor changes in chronic stroke survivors who were administered mental practice. Archives of Physical Medicine and Rehabilitation, 92(11), 1741-1745. doi: 10.1016/j.apmr.2011.06.009

Papaxanthis, C., Schieppati, M., Gentili, R., \& Pozzo, T. (2002). Imagined and actual arm movements have similar durations when performed under different conditions of direction and mass. Experimental Brain Research, 143(4), 447-452.

Perfetti, C., \& Rossetto, F. (1997). L'immagine motoria come elemento dell'esercizio terapeutico conoscitivo. Ipotesi preliminary. Riabilitazione e Apprendimento, 2, 109-116.

Sabate, M., Gonzalez, B., \& Rodriguez, M. (2004). Brain lateralization of motor imagery: Motor planning asymmetry as a cause of movement lateralization. Neuropsychologia, 42(8), 1041-1049. doi: 10.1016/j.neuropsychologia.2003.12.015

Sharma, N., Pomeroy, V. M., \& Baron, J. C. (2006). Motor imagery: A backdoor to the motor system after stroke? Stroke, 37(7), 1941-1952.

Sterr, A., \& Conforto, A. B. (2012). Plasticity of adult sensorimotor system in severe brain infarcts: Challenges and opportunities. Neural Plasticity, 2012, 970136. doi: 10.1155/2012/970136

Stevens, J. A., \& Stoykov, M. E. (2003). Using motor imagery in the rehabilitation of hemiparesis. Archives of Physical Medicine \& Rehabilitation, 84(7), 1090-1092.

Stinear, C. M., Fleming, M. K., Barber, P. A., \& Byblow, W. D. (2007). Lateralization of motor imagery following stroke. Clin Neurophysiol, 118(8), 1794-1801. doi: 10.1016/j.clinph.2007.05.008

Szameitat, A. J., Shen, S., Conforto, A., \& Sterr, A. (2012). Cortical activation during executed, imagined, observed, and passive wrist movements in healthy volunteers and stroke patients. Neuroimage, 62(1), 266-280. doi: 10.1016/j.neuroimage.2012.05.009

Varkuti, B., Guan, C., Pan, Y., Phua, K. S., Ang, K. K., Kuah, C. W., Sitaram, R., et al. (2013). Resting state changes in functional connectivity correlate with movement recovery 
for BCI and robot-assisted upper-extremity training after stroke. Neurorehabilitation and Neural Repair, 27(1), 53-62. doi: $10.1177 / 1545968312445910$

Warner, L., \& McNeill, M. E. (1988). Mental imagery and its potential for physical therapy. Physical Therapy, 68(4), 516-521.

Wolbers, T., Weiller, C., \& Buchel, C. (2003). Contralateral coding of imagined body parts in the superior parietal lobe. Cerebral Cortex, 13(4), 392-399.

Yaguez, L., Nagel, D., Hoffman, H., Canavan, A. G., Wist, E., \& Homberg, V. (1998). A mental route to motor learning: Improving trajectorial kinematics through imagery training. Behavioural Brain Research, 90(1), 95-106.

Yoo, E., Park, E., \& Chung, B. (2001). Mental practice effect on line-tracing accuracy in persons with hemiparetic stroke: A preliminary study. Archives of Physical Medicine and Rehabilitation, 82(9), 1213-1218.

Yue, G., \& Cole, K. J. (1992). Strength increases from the motor program: Comparison of training with maximal voluntary and imagined muscle contractions. Journal of Neurophysiology, 67(5), 1114-1123. 